\title{
Impact of the COVID-19 Pandemic on the Appropriateness of Diagnostic Pathways of Autoimmune Rheumatic Diseases
}

\author{
Teresa Carbone ${ }^{1} \mathbb{D}$, Valentina Picerno ${ }^{2}$, Vito Pafundi ${ }^{3}$, Ernesto Esposito ${ }^{4}$, Pietro Leccese ${ }^{2}$ (D), \\ Angela Anna Padula², and Salvatore D’Angelo ${ }^{2}$ (i)
}

\begin{abstract}
Objective. Early diagnosis of autoimmune rheumatic diseases (ARDs) is key to achieving effective treatment and improving prognosis. The coronavirus disease 2019 (COVID-19) pandemic has led to major changes in clinical practice on a global scale. We aimed to evaluate the impact of the COVID-19 pandemic on rheumatological clinical practice and autoimmunity testing demands.

Methods. Data regarding the first rheumatological visits and new diagnoses, together with the autoimmunity laboratory testing volumes related to the COVID-19 pandemic phase (January-December 2020), were collected from medical records and the laboratory information system of a regional reference hospital (Basilicata, Italy) and compared with those obtained during the corresponding period in 2019.

Results. A significant decrease in the 2020 autoimmunity laboratory test volume was found when compared with the same period in 2019 (9912 vs 14,100; $P<0.05$ ). A significant decrease in first rheumatological visits and diagnosis (1272 vs 2336; $P<0.05$ ) was also observed. However, an equivalent or higher percentage of positive autoimmunity results from outpatient services was recorded during 2020 when compared to the prepandemic state. Of note, COVID-19-associated decline in new diagnoses affected mainly less severe diseases. In contrast, ARDs with systemic involvement were diagnosed at the same levels as in the prepandemic period.

Conclusion. The COVID-19 pandemic has affected access to health services. However, our study highlighted that during the outbreak, greater appropriateness of the requests for laboratory tests and visits emerged, as shown by a greater percentage of positive test results and new diagnoses of more severe ARDs compared to the prepandemic period.
\end{abstract}

Key Indexing Terms: autoimmune rheumatic diseases, autoimmunity, autoimmunity test, COVID-19, impact, rheumatology

Coronavirus disease 2019 (COVID-19) is a newly discovered systemic, infectious viral disease caused by severe acute respiratory syndrome coronavirus 2 (SARS-CoV-2). The first human case was reported in December 2019 in Wuhan, China. Given the alarming rate of spread and the severity of the disease, COVID-19 was declared a public health emergency of international concern on January 30, 2020, and a pandemic on March 11, 2020, by the World Health Organization. ${ }^{1}$ As of Week 30 in 2021, almost 34 million confirmed COVID-19 cases have been reported in the European Union/European Economic Area,

${ }^{I}$ T. Carbone, PhD, Laboratory of Clinical Pathology, Azienda Sanitaria di Matera (ASM), Matera; ${ }^{2}$ V. Picerno, MD, P. Leccese, MD, A.A. Padula, $M D$, S. D'Angelo, MD, PhD, Rheumatology Institute of Lucania - San Carlo Hospital, Potenza; ${ }^{3}$ V. Pafundi, MD, Immunopathology Laboratory, San Carlo Hospital, Potenza; ${ }^{4}$ E. Esposito, MD, Health Department, Basilicata Region, Potenza, Italy.

T. Carbone and V. Picerno contributed equally.

The authors declare no conflict of interests relevant to this article.

Address correspondence to Dr. V. Pafundi, Immunopathology Laboratory,

San Carlo Hospital, Potito Petrone St., 85100 Potenza, Italy.

Email:vito.pafundi@ospedalesancarlo.it.

Accepted for publication September 30, 2021. with 745,014 deaths. ${ }^{2}$ The COVID-19 pandemic has challenged the resilience of health systems of all the world. ${ }^{3}$

On February 20, 2020, the first severe case of SARS-CoV-2related pneumonia was diagnosed in northern Italy. Since then, the COVID-19 pandemic has rapidly affected all Italian regions. The first wave initiated in March 2020 and lasted for 3 months, requiring home confinement and strict lockdown. Following the relaxation of measures during the summer, a second wave commenced in September 2020 and extended until December 2020. Basilicata is a small southern region of Italy that counts 562,869 inhabitants $\left(56 / \mathrm{km}^{2}\right), 22.3 \%$ of whom are aged $>65$ years. ${ }^{4}$ From the beginning of the emergence of the COVID-19 pandemic until December 2020, a total of 117,865 subjects underwent SARS-CoV-2 testing in Basilicata, with 184,291 SARS-CoV-2 swab tests performed overall and 12,987 positive results recorded. ${ }^{5}$ San Carlo Hospital was rapidly overwhelmed and there was a reallocation of most of the available healthcare resources, both to treat patients with COVID-19 and to process SARS-CoV-2 swabs, leading to unprecedented changes in clinical practice. Rheumatology services have been reconfigured and medical staff redeployed to the front line. During the lockdown (March-May 2020), according to ministerial directives, routine rheumatology ambulatory activities were suspended and all 
nonurgent outpatient clinical visits were cancelled. In order to prevent transmission of the viral infection, the rapid deployment of telemedicine use was adopted in the ambulatory services. Teleconsultation was introduced as an innovative channel of communication, allowing for a reduction of face-to-face consultations while at the same time benefiting the rheumatic patient. ${ }^{6}$

Increasing local incidence of COVID-19 also had a profound effect on diagnostic activity of the autoimmunity laboratory, requiring the complete reorganization of human resources and procedures. Whereas volume increases for laboratory tests related to COVID-19 diagnostics were observed, autoimmunity testing volume decreased worldwide. ${ }^{7}$ Autoantibodies are key serological markers to screen for and support the diagnosis of autoimmune rheumatological diseases (ARDs). ${ }^{8,9,10,11,12}$ As early diagnosis of ARDs is important in order to improve longterm outcomes, the situation has been extremely challenging for laboratory staff, clinical rheumatologists, and rheumatological patients themselves. ${ }^{13,14,15,16}$

Data regarding the effect of the COVID-19 outbreak on the number of autoimmunity tests performed and on rheumatological diagnoses of ARD are lacking. Understanding the effects of the pandemic on specific areas of health care is crucial for future planning in both the immediate and longer term.

Based on these premises, the aims of this study are to evaluate (1) the changes in the demand for autoimmunity testing assayed at the San Carlo Hospital Immunopathology Laboratory and the percentage of positive results; and (2) the number of rheumatological visits and new ARD diagnoses carried out at the Rheumatological Institute of Lucania of San Carlo Hospital in Potenza, Italy in the setting of increasing local incidence of COVID-19.

\section{METHODS}

Data collection. The San Carlo Hospital, located in the regional capital, Potenza, is the largest hospital in the Basilicata region and the leading local center for ARDs. To report the effect of the COVID-19 pandemic on our practice, we analyzed clinical and laboratory activity trends comparing 2 distinct periods: from January to December of 2020 and the same period in 2019. All first rheumatological visits and follow-up visits specifically relating to flares were carried out in person, while all other follow-up visits were done by teleconsultation.

The diagnosis of each patient was made by an expert rheumatologist on the basis of history, physical examination, blood, and serological tests and registered in the electronic medical chart. Data were extracted by 2 independent rheumatologists (V. Picerno and PL) and new diagnoses of rheumatic diseases were categorized as follows: rheumatoid arthritis (RA), psoriatic arthritis (PsA), axial (ax-) spondyloarthritis ( $\mathrm{PpA}$ ), other $\mathrm{SpA}$, systemic lupus erythematosus (SLE), systemic sclerosis (SSc), Sjögren syndrome (SS), polymyositis/dermatomyositis (PM/DM), undifferentiated connective tissue disease/mixed connective tissue disease (UCTD/MCTD), antiphospholipid syndrome (APS), osteoarthritis (OA), microcrystalline arthropathies, osteoporosis (OP), fibromyalgia (FM), juvenile idiopathic arthritis (JIA), polymyalgia rheumatica (PMR), Behçet disease (BD), vasculitis, and other diseases (regional pain syndrome [RPS], skin diseases without systemic involvement, peripheral neuropathies).

Data on autoimmunity laboratory tests such as antinuclear antibodies (ANA), anti-extractable nuclear antigen (ENA) antibodies, anti-dsDNA antibodies, rheumatoid factor $(\mathrm{RF})$, anticitrullinated protein antibodies (ACPA), and antineutrophil cytoplasmic antibodies (ANCA), including myeloperoxidase (MPO) and proteinase 3 (PR3) antibodies, were collected from the laboratory information system (LIS). A filter was also applied to select samples coming only from outpatient services. During the observation period of 2020, outpatient testing volumes were calculated and used as reference points to examine the absolute and relative differences in test volume compared to the prepandemic period.

When outpatient autoimmunity tests were selected for the analysis, positive results for each serological marker were extracted from LIS and the absolute and relative (\%) differences in prepandemic and pandemic periods were calculated in order to assess the overall changes. In addition, we analyzed the percentage of positive findings on the total volume of outpatient testing.

Data collection was entirely retrospective and did not involve any direct patient contact (registration number at Common Regional Ethics Committee of Basilicata [CEUR]: 56/2021).

Statistical analysis. Total laboratory testing volume, rheumatological visits, and new diagnoses during the observation periods (January-December 2019 vs 2020) were calculated. Absolute and relative differences (\%) between the prepandemic and pandemic periods were also calculated. Continuous variables were analyzed using unpaired $t$ tests or Mann-Whitney $U$ tests, and categorical variables were analyzed using chi-square tests or Fisher exact tests when appropriate. All statistical analyses were carried out using SAS v 9.2 software (SAS Institute). A $P$ value $<0.05$ was considered statistically significant.

\section{RESULTS}

Impact of the COVID-19 pandemic on autoimmunity laboratory testing orders. We first analyzed the effect of the pandemic on autoimmunity testing orders coming from both outpatients and inpatients, and a statistically significant reduction of all investigated laboratory parameters was found during the pandemic period. In total, 9912 and 14,100 tests were performed in 2020 and 2019 , respectively. In the pandemic period, these tests were ordered by different health professionals: general practitioners (GPs; 70\%), rheumatologists (10\%), and other specialists (20\%). There were nearly 1.5 times fewer tests ordered for ANA, anti-ENA, anti-dsDNA, RF, ANCA, and ACPA in 2020 compared to 2019 ( $P<0.05$ for all comparisons; data not shown).

Impact of the COVID-19 pandemic on autoimmunity laboratory tests coming from outpatients. All laboratory tests coming from outpatients showed the same trend, with a strong decline during the first wave and a smaller decrease during the second wave of the COVID-19 pandemic, remaining lower than the prepandemic period for the entire year (Figure 1A-F).

Compared with the same period in 2019, there were roughly 1.5 times fewer samples taken from outpatients for ANA, anti-ENA, anti-dsDNA, RF, ANCA, and ACPA testing during the 2020 pandemic period $(P<0.05)$

We found an initial sustained reduction in test volume during March 2020, reaching a peak in April: -95\% ANA, $-93 \%$ anti-ENA, $-94 \%$ anti-dsDNA, $-96 \%$ RF, -97\% ACPA, and $-85 \%$ ANCA (Figure 1A-F).

Impact of the COVID-19 pandemic on autoimmunity laboratory-positive findings. A statistically significant reduction in the absolute number of positive ANA and RF tests was found in the 2020 study period when compared to 2019 (ANA: 552 vs 730; RF: 202 vs 275; $P<0.05)$. On the contrary, no significant change was observed between 2020 and 2019 when positive findings for ENA, anti-dsDNA, ACPA, and ANCA testing were 


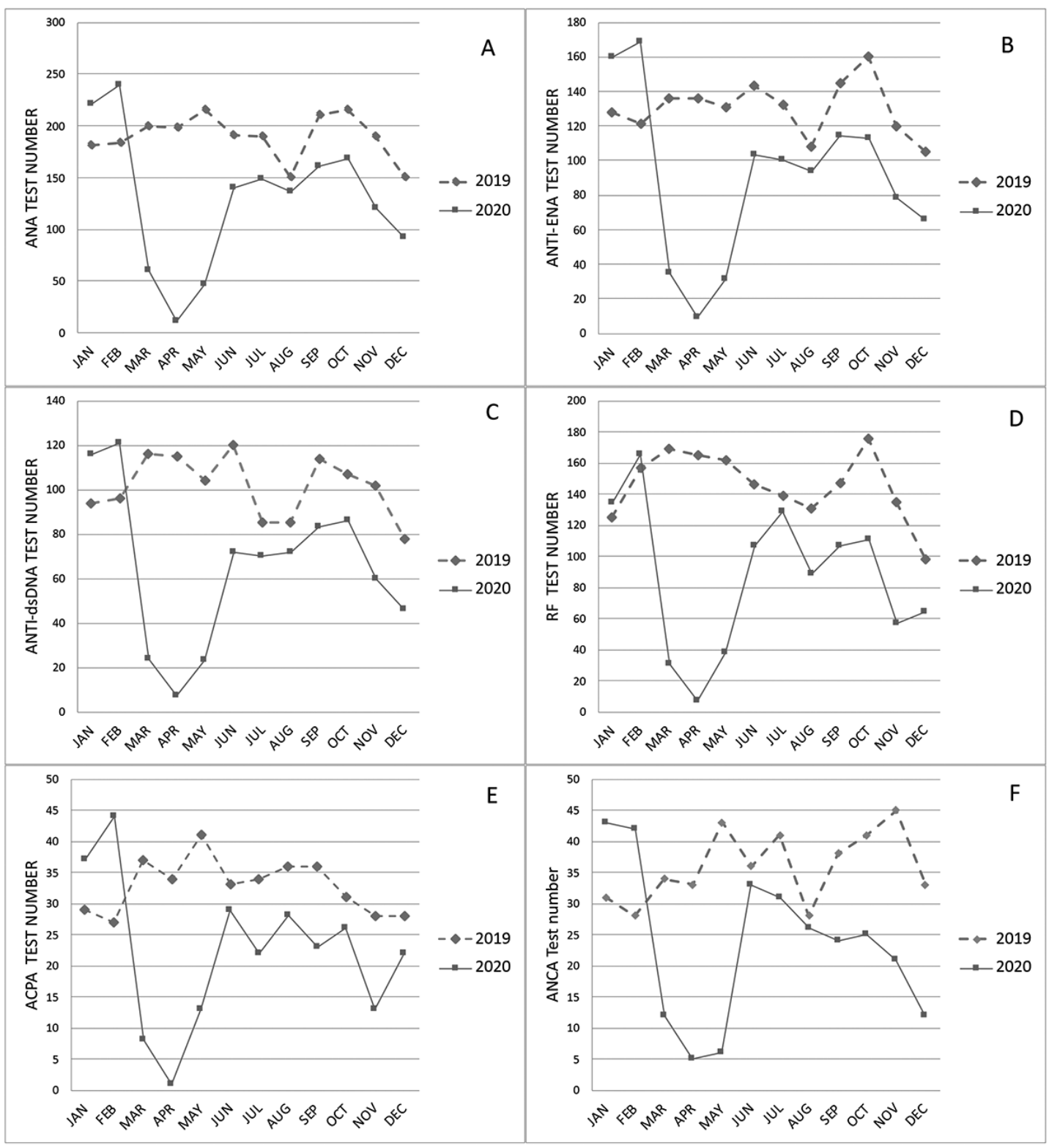

Figure 1. Autoimmunity laboratory test order trends. Autoimmunity laboratory test orders from outpatients plotted as a function of time from January to December. For each analyte (A-F) data for 2020 were compared with the same period for 2019. ACPA: anticitrullinated protein antibodies; ANA: antinuclear antibody; ANCA: antineutrophil cytoplasmic antibodies; ENA: extractable nuclear antigens; RF: rheumatoid factor.

evaluated (ENA: 131 vs 179; anti-dsDNA: 15 vs 12; ACPA: 43 vs 60; ANCA: 12 vs 14 ).

A higher percentage of positive autoimmunity results was observed during the pandemic period, especially for ANA, anti-dsDNA, RF, and ANCA testing (Figure 2). It is worth noting that during the lockdown (March-May 2020), greater differences were observed. In particular, the percentage of ANA-positive results was $44.0 \%$ compared to $31.4 \%$ reported in the same period of 2019, whereas anti-dsDNA positivity was $1.9 \%$ vs $1.5 \%$, RF positivity was $19.7 \%$ vs $15.1 \%$, and ANCA positivity was $4.3 \%$ vs $0.9 \%$ (data not shown).

Impact of COVID-19 on access to rheumatological visits and diagnoses. Compared with 2019, a significant reduction in first rheumatological visits and new diagnoses was observed in 2020 (Figure 3). The absolute difference between 2020 and 2019 study periods was equal to 1064 rheumatological visits (1272 vs 2336), with a decrease in total visits of $48 \%$.

As shown in Figure 3, the highest percentage change was recorded during the lockdown period (March-May 2020), in which total visits decreased by $83 \%$.

In particular, a marked reduction emerged for new diagnoses of less severe conditions and noninflammatory diseases, such as OA, FM, and RPS (Figure 4). Comparative analysis between new diagnoses on the total number of visits for year was also carried out. A statistically significant reduction emerged for new diagnoses of PsA, SSc, OA, microcrystalline arthropathies, 


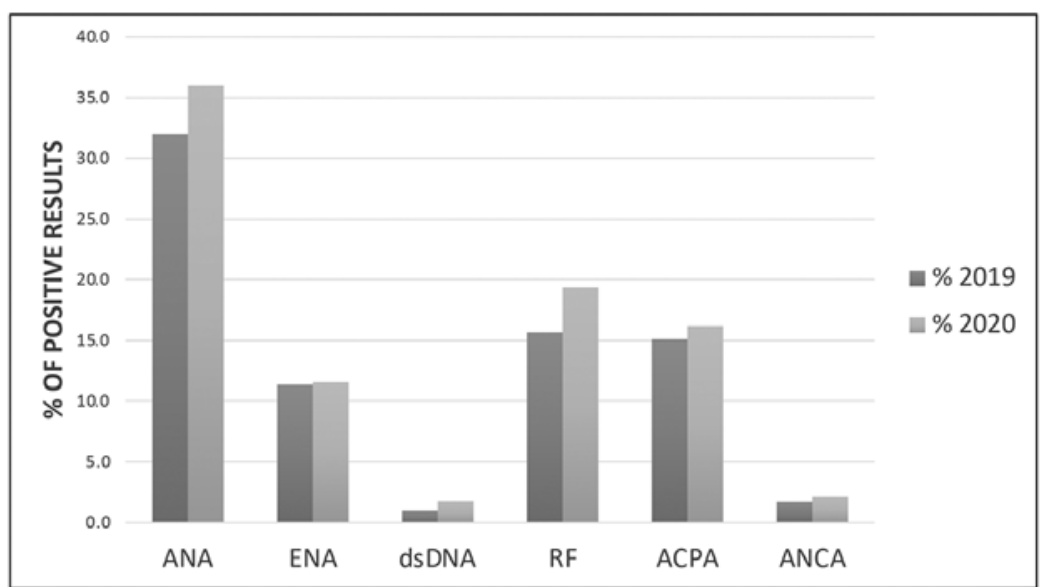

Figure 2. Autoimmunity laboratory-positive findings. Relative differences in percentage of positive results for each marker between prepandemic and pandemic periods. ACPA: anticitrullinated protein antibodies; ANA: antinuclear antibody; ANCA: antineutrophil cytoplasmic antibodies; ENA: extractable nuclear antigens; RF: rheumatoid factor.

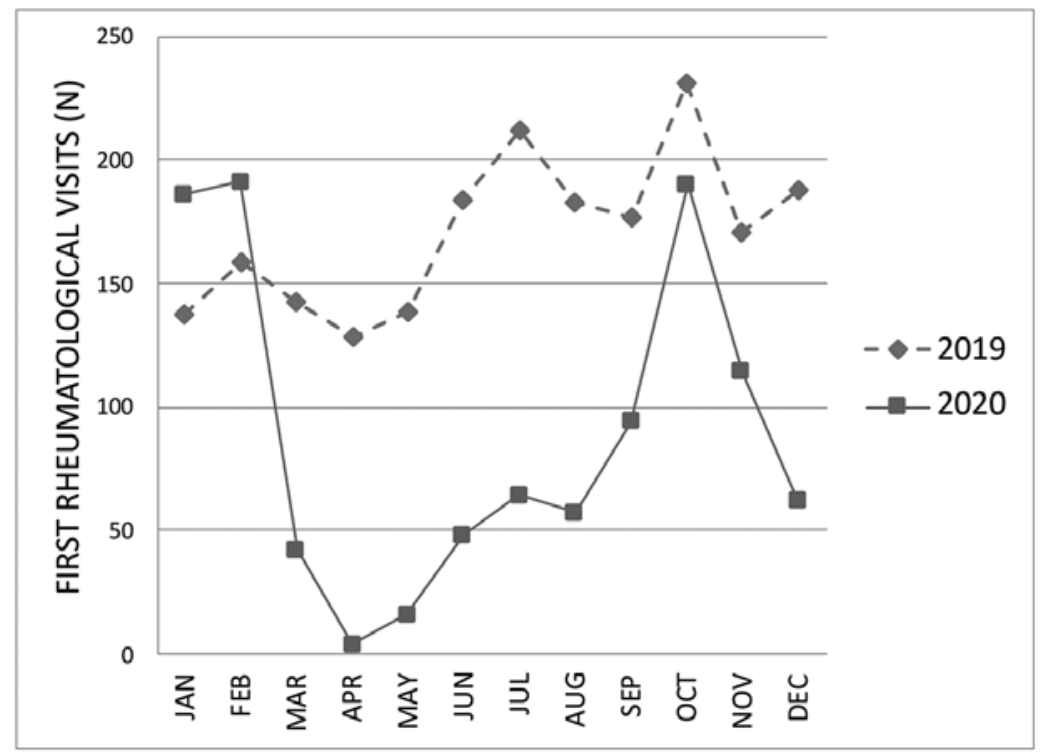

Figure 3. First rheumatological visit trends, plotted as a function of time from January to December for 2020 vs 2019.

OP, FM, PMR, and other diseases $(P<0.05)$, with a substantial increase for SLE and SS diagnosis $(P<0.05)$. In contrast, for RA, axSpA, PsA, other SpA, PM/DM, UCTD/MCTD and APS, JIA, BD, and vasculitis, the number of new diagnoses did not undergo significant change (Figure 4).

\section{DISCUSSION}

To our knowledge, this is the first study describing the effect of the COVID-19 pandemic on rheumatological clinical practice and on autoimmunity testing demand. Our results demonstrated that, even in the presence of a serious ongoing emergency, patients with high clinical suspicion of severe rheumatic disease have benefited from both autoimmunity tests and rheumatologist visits to receive a proper diagnosis.

The COVID-19 outbreak has rapidly affected hospitals worldwide, resulting in a rapid and forced reorganization of human resources and activities. Additionally, this has created a greater challenge for the management of patients affected by other diseases. ${ }^{16,17}$ Some effects of the COVID-19 pandemic on healthcare systems have been investigated ${ }^{13,18-23}$; limited data are available on the effects on management of rheumatic diseases, ${ }^{6,15,16,24}$ but no study has addressed its influence on appropriateness of diagnostic pathways of autoimmune diseases.

For the diagnosis and follow-up of ARDs, an integrated approach based on clinical and laboratory criteria is recommended. An early diagnosis can ensure appropriate treatment to avoid systemic complications and improve disease prognosis. ${ }^{10,11,12,25,26}$

In line with other studies, ${ }^{22,27,28}$ we recorded a decrease in autoimmunity test volume coming from both outpatients and inpatients with an absolute difference of $4188(-29.7 \%)$ between 2019 and 2020. In particular, when outpatient testing 


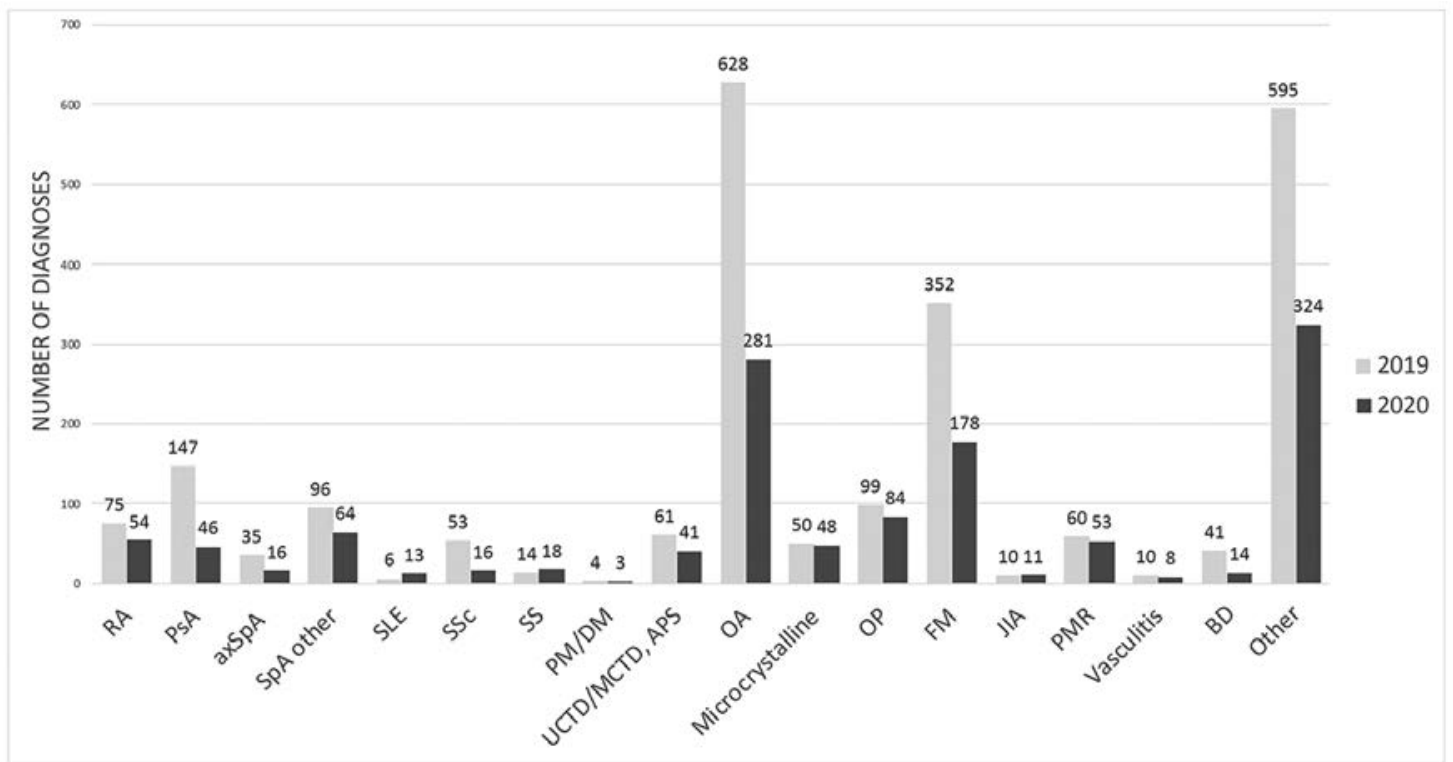

Figure 4. The effect of the COVID-19 pandemic on new diagnoses of rheumatic diseases. The bar graph shows the volume of diagnoses of rheumatic diseases carried out between January and December of 2019 and 2020. The respective values are reported at the top of the columns. APS: antiphospholipid syndrome; axSpA: axial spondyloarthritis; BD: Behçet disease; FM: fibromyalgia; JIA: juvenile idiopathic arthritis; Microcrystalline: microcrystalline arthropathies; OA: osteoarthritis; OP: osteoporosis; PM/DM: polymyositis/dermatomyositis; PMR: polymyalgia rheumatica; PsA: psoriatic arthritis; RA: rheumatoid arthritis; SLE: systemic lupus erythematosus; SpA other: spondyloarthritis other than axSpA and PsA; SS: Sjögren syndrome; SSc: systemic sclerosis; UCTD/ MCTD: undifferentiated connective tissue disease/mixed connective tissue disease.

was considered, a reduction of $32.1 \%$ was observed for ANA testing samples, $31.5 \%$ for anti-ENA, $35.9 \%$ for anti-dsDNA, $40.7 \%$ for RF, $35.1 \%$ for ACPA, and 32.5\% for ANCA. A significant drop was also observed for first rheumatological visits, with an absolute difference of $1064(-48.0 \%)$ and the highest decline observed between March and May 2020 (-83\%).

These results can be explained by considering patients' attitudes (e.g., patients may be less likely to seek services for nonurgent care to avoid perceived higher risk of COVID-19 infection in a healthcare setting) and hospital policies to postpone nonurgent visits during lockdown. Indeed, during the pandemic period the reorganization of hospital logistics and clinical procedures caused a forced reduction in inpatient and outpatient services provided to non-COVID patients.

When positive results of autoimmunity testing were extrapolated for the study periods, an interesting finding emerged: while the absolute number of tests decreased, the percentage of positive results on total volume increased. In particular, during the period between March and May, the positive rate for ANA testing was $44.0 \%$ in 2020 vs $31.4 \%$ in $2019 ; 1.9 \%$ vs $1.5 \%$ for anti-dsDNA; $19.7 \%$ vs $15.1 \%$ for RF; and 4.3 vs $0.9 \%$ for ANCA. These results suggest an increase in the appropriateness and specificity of requests of autoimmunty testing by clinicins during the pandemic.

Regarding appropriateness, according to the Agency for Healthcare Research and Quality, "the clinical utility of medical tests is measured by whether the information they provide affects patient-relevant outcomes, ${ }^{\prime 29}$ and it depends on several variables such as clinical context, severity of the diseases, administrative necessities, pressure to test from patients, fear of litigation, inexperience or impatience of physician, etc. ${ }^{30,31,32}$

During the pandemic, we believe the most likely reason for the testing decline was that many patients chose to manage nonurgent conditions by themselves. Therefore, only tests with greater pretest clinical suspicion were requested by clinicians.

In this setting, data regarding the volume of new ARD diagnoses were also analyzed and a significant reduction was found during the COVID-19 pandemic compared to the same period in 2019. A significant decrease was specifically observed for noninflammatory conditions or diseases without systemic involvement, such as OA, OP, FM, RPS, and peripheral neuropathies. In contrast, no significant changes were observed in the number of new diagnoses of chronic inflammatory rheumatic diseases characterized by more severe features at onset. However, a slight reduction in new diagnoses of PsA, SSc, and BD was observed. This may be due to containment measures and restrictions placed on people's movement during the lockdown. Indeed, the San Carlo Hospital is a leading center for such diseases and attracts patients from other regions of Southern Italy.

Our study has some limitations. First, this is mainly a descriptive study with data reflecting experience from a single center. In addition, given the central role of primary care services during emergencies, GPs were called to manage a growing number of health situations as demonstrated by the high percentage of first rheumatological visits (70\% of the total number) referred by GPs in our study. In this context, the varying rheumatological skills of GPs could influence both the ordering of laboratory tests and the referral to rheumatologists. We were not able to explore the 
involvement of different GPs in the 2 study periods and their contributions to improving the appropriateness of the ordered tests. In addition, we were unable to intercept very severe cases that were brought directly to emergency departments, such as connective tissue diseases and vasculitis with life-threatening manifestations. Finally, the possible influence of asymptomatic COVID-19 infections on autoimmunity response in terms of autoantibody production was not evaluated. Despite these limitations, our study provides important insights regarding the appropriateness of autoimmunity laboratory testing and outcomes of prioritization strategies for face-to-face rheumatological visits developed during the COVID-19 outbreak.

Our data could be useful to better manage the appropriateness of the ARD diagnostic pathway in daily clinical practice as well as in a future pandemic setting.

In conclusion, although there were challenges for clinical practice during the COVID-19 pandemic, safe and high-quality healthcare was maintained for patients with severe ARDs.

\section{REFERENCES}

1. World Health Organization. News and media: virtual press conference on COVID-19 - 11 March 2020. [Internet. Accessed October 13, 2021.] Available from: https://www.who.int/docs/ default-source/coronaviruse/transcripts/who-audio-emergenciescoronavirus-press-conference-full-and-final-1 1 mar2020.pdf

2. European Centre for Disease Prevention and Control COVID-19. [Internet. Accessed October 7, 2021] Available from: https://www. ecdc.europa.eu/en/covid-19

3. Blumenthal D, Fowler EJ, Abrams M, Collins SR. Covid-19implications for the health care system. N Engl J Med 2020;383:1483-8.

4. I.Stat. Resident population on 1 January: Basilicata. [Internet. Accessed October 7, 2021] Available from: https://dati.istat.it/ Index.aspx ?QueryId=18564

5. Regione Basilicate. [Covid-19 emergency, update December 31 (Data 30/12)] [Article in Italian]. [Internet. Accessed October 7, 2021] Available from: https://www.regione.basilicata.it/giunta/site/ Giunta/detail.jsp?otype $=1012 \&$ id $=3071571$

6. Mühlensiepen F, Kurkowski S, Krusche M, et al. Digital health transition in rheumatology: a qualitative study. Int J Environ Res Public Health 2021;18:2636.

7. Durant TJS, Peaper DR, Ferguson D, Schulz WL. Impact of COVID-19 pandemic on laboratory utilization. J Appl Lab Med 2020;5:1194-1205.

8. Bossuyt X, De Langhe E, Borghi MO, Meroni PL. Understanding and interpreting antinuclear antibody tests in systemic rheumatic diseases. Nat Rev Rheumatol 2020;16:715-26.

9. Carbone T, Gilio M, Padula MC, Tramontano G, D’Angelo S, Pafundi V. A step towards standardization: a method for end-point titer determination by fluorescence index of an automated microscope. End-point titer determination by fluorescence index. J Immunol Methods 2018;456:67-71.

10. Carbone T, Esposito C, Pafundi V, et al. Understanding the biological significance of anti-DFS70 antibodies: effect of biologic therapies on their occurrence in inflammatory arthritis. J Rheumatol 2020;47:1295-6.

11. Carbone T, Pafundi V, Bizzaro N, et al. Assessing vitamin D levels in an anti-DFS70 positive population: new insights emerging. Autoimmunity 2020;53:231-6.

12. Infantino M, Carbone T, Manfredi M, et al. Are anti-DFS70 autoantibodies protective? Isr Med Assoc J 2019;21:509-11.

13. Zhang YN, Chen Y, Wang Y, et al. Reduction in healthcare services during the COVID-19 pandemic in China. BMJ Glob Health 2020;5:e003421.
14. Moynihan R, Sanders S, Michaleff ZA, et al. Impact of COVID-19 pandemic on utilisation of healthcare services: a systematic review. BMJ Open 2021;11:e045343.

15. Ciurea A, Papagiannoulis E, Bürki K, et al. Impact of the COVID-19 pandemic on the disease course of patients with inflammatory rheumatic diseases: results from the Swiss Clinical Quality Management cohort. Ann Rheum Dis 2021;80:238-41.

16. Dejaco C, Alunno A, Bijlsma JW, et al. Influence of COVID-19 pandemic on decisions for the management of people with inflammatory rheumatic and musculoskeletal diseases: a survey among EULAR countries. Ann Rheum Dis 2021;80:518-26.

17. Gruber A, Ghiringhelli M, Edri O, et al. Literature review and knowledge distribution during an outbreak: a methodology for managing infodemics. Acad Med 2021;96:1005-9.

18. Kim Y, Ahn E, Lee $S$, et al. Changing patterns of medical visits and factors associated with no-show in patients with rheumatoid arthritis during COVID-19 pandemic. J Korean Med Sci 2020;35:e423.

19. Alboksmaty A, Kumar S, Parekh R, Aylin P. Management and patient safety of complex elderly patients in primary care during the COVID-19 pandemic in the UK-Qualitative assessment. PLoS One 2021;16:e0248387.

20. Kruizinga MD, Peeters D, van Veen M, et al. The impact of lockdown on pediatric ED visits and hospital admissions during the COVID19 pandemic: a multicenter analysis and review of the literature. Eur J Pediatr 2021;180:2271-9.

21. Frater JL, Zini G, d'Onofrio G, Rogers HJ. COVID-19 and the clinical hematology laboratory. Int J Lab Hematol 2020;42 Suppl 1:11-8.

22. Pellegrini M, Roda M, Lupardi E, Di Geronimo N, Giannaccare G, Schiavi C. The impact of COVID-19 pandemic on ophthalmological emergency department visits. Acta Ophthalmol 2020;98:e1058-9.

23. Dopfer C, Wetzke M, Zychlinsky Scharff A, et al. COVID-19 related reduction in pediatric emergency healthcare utilization - a concerning trend. BMC Pediatr 2020;20:427.

24. Roux CH, Brocq O, Gerald F, Pradier C, Bailly L. Impact of home confinement during the COVID-19 pandemic on medication use and disease activity in spondyloarthritis patients. Arthritis Rheumatol 2020;72:1771-2.

25. Bizzaro N. Autoantibody profiles in autoimmune rheumatic diseases. Mediterr J Rheumatol 2019;30:86-9.

26. Tozzoli R, Bizzaro N. The clinical and the laboratory autoimmunologist: where do we stand? Auto Immun Highlights 2020;11:10.

27. Earnshaw CH, Hunter HJA, McMullen E, Griffiths CEM, Warren RB. Reduction in skin cancer diagnosis, and overall cancer referrals, during the COVID-19 pandemic. Br J Dermatol 2020;183:792-4.

28. Minucci A, Scambia G, Santonocito C, Concolino P, Urbani A. BRCA testing in a genomic diagnostics referral center during the COVID-19 pandemic. Mol Biol Rep 2020;47:4857-60.

29. Trikalinos TA, Siebert U, Lau J. Decision-analytic modeling to evaluate benefits and harms of medical tests: uses and limitations. Med Decis Making 2009;29:E22-9.

30. Cappelletti P. Appropriateness of diagnostics tests. Int J Lab Hematol 2016;38 Suppl 1:91-9.

31. Ferraro S, Panteghini M. The role of laboratory in ensuring appropriate test requests. Clin Biochem 2017;50:555-61.

32. Sack U, Bossuyt $\mathrm{X}$, Andreeva $\mathrm{H}$, et al; European Autoimmunity Standardisation Initiative. Quality and best practice in medical laboratories: specific requests for autoimmunity testing. Auto Immun Highlights 2020;11:12. 